\title{
A rare case of thoracic diaphragmatic endometriosis invading the liver and lower lobe of the lung
}

\author{
Azal EL Adwan, Harjit Singh Dhaliwal, Samah Ballal, Rachana Dwivedi
}

\author{
Corresponding author: Dr Harjit Singh Dhaliwal, Department of Obstetrics and Gynaecology , \\ Royal Bournemouth Hospital, Castle Lane East , Bournemouth , BH7 7DW \\ Email - harjitdhaliwal2004@yahoo.co.uk
}

Distributed under Attribution-Non Commercial - Share Alike 4.0 International (CC BY-NC-SA 4.0)

\begin{abstract}
We present a case of a 47 year old female who was referred to the respiratory physicians with a cough and haemoptysis. She has also been having periodic right upper quadrant pain as well as right arm pain for the last 15 years. She had been seen her General Practitioner on numerous occasions in the past without a ruling diagnosis and resolution of her symptoms. CT scan suggested a mass invading the right hemi-diaphragm, lower lung and the right lobe of the liver. Liver biopsy suggested small cores of benign endometrial glands and stroma-endometriosis.
\end{abstract}

Keywords: Endometriosis, diaphragm, pleura, haemoptysis, right upper quadrant pain, shoulder pain.

Endometriosis is a disease characterised by the presence of normal endometrial tissue abnormally implanted in locations other than the uterine cavity. Depending on the place of implantation, endometriosis can be expressed in a wide variety of clinical symptoms. It is commonly found in the pelvis ( ovaries, uterine ligaments, Pouch of Douglas and fallopian tubes), however it may be found in extrapelvic locations such as; gastrointestinal tract (GI), brain, liver , lungs, urinary tract, abdominal wall and viscera ${ }^{1}$.

Endometriosis affects about $3-10 \%$ of the reproductive age group, with an estimated $12 \%$ of these patients have extragenital endometriosis ${ }^{2}$. It also affects $2-5 \%$ of postmenopausal women. Functioning endometrial tissue endometriosis can involve the diaphragm, pleura, lung parenchyma and more rarely in the tracheobronchial tree. The thoracic diaphragm (38.8\%) and visceral diaphragm $(29.6 \%)$ are the most common sites of lesions with the lung parenchyma being less commonly reported ${ }^{3}$. In a case series by Nezhat et al they found that $76 \%$ of the patients had both thoracic and visceral involvement with only a small proportion having either the thoracic or visceral component. They also found a higher proportion of women with lung parenchymal involvement (40\%). The authors also reported that with thoracic endometriosis there was a $100 \%$ concurrent pelvic endometriosis compared with previous citations of $50-80 \%{ }^{4}$. Hence, there should be a high index of suspicion based on history and examination of endometriosis in preparation for further management.

Extrapelvic endometriosis is a rare and a complex pathology. Endometriosis involving the thorax - thoracic endometriosis syndrome, is a rare manifestation of extragenital endometriosis with important clinical ramifications. Despite growing awareness of thoracic endometriosis, it remains an enigmatic condition with various theories proposed to explain its intrathoracic presence. The diagnosis of thoracic endometriosis depends

Received: $24^{\text {th }}$ June 2019. Accepted: $19^{\text {th }}$ September 2019.

Adwan AEL, Dhaliwal HS, Ballal S, Dwivedi R. A rare case of thoracic diaphragmatic endometriosis invading the liver and lower lobe of the lung. The New Indian Journal of OBGYN. 2020; 7(1): 97-101. 
heavily on clinical suspicion, as diagnosis is often delayed. It could mean that the thoracic component takes a longer time to develop or it being underdiagnosed due to the lack of awareness amongst clinicians. This is solely based on the lack of a detailed history taken at the time of consultation. Depending on the place of tissue implantation, endometriosis can present with a wide variety of symptoms. Hence, there should be a high index of clinical suspicion in every woman of periodic symptoms of extragenital organs.

\section{Case}

A 47 year old presented to the Fast Track chest clinic with cough of 10 months duration, which was intermittently productive of small amounts of brown to red haemoptysis. Interestingly, she did wonder if her haemoptysis coincided with her menstruation. She also reported having had right upper quadrant pain and ipsilateral right shoulder and arm pain for approximately 15 years. This also coincided with her menstruation.

A

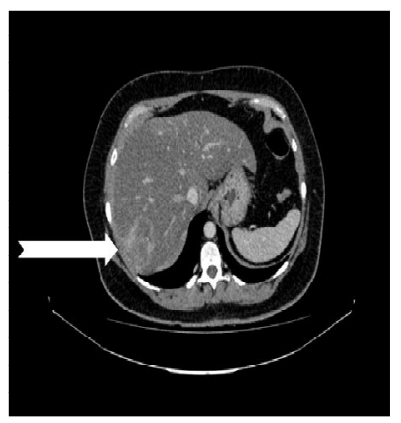

B

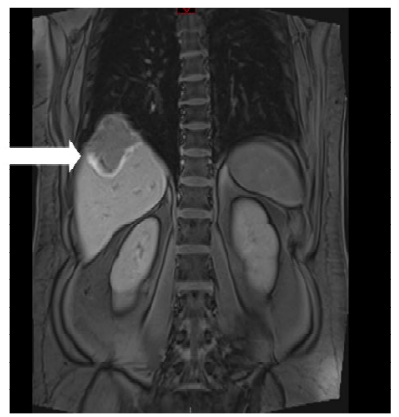

Figure 1: $C T$ Scan and MRI on presentation. A) CT scan showing mass invading the right hemidiaphragm into right lobe of the liver. B) MRI showing The lesion spans the right hemidiaphragm posteriorly into the liver and the right lower lobe of the lung. The liver lesion suggests haemorrhage and may reflect a haematoma.

She has been an ex-smoker having smoked approximately a total of 15 pack years on and off. She stopped when these problems arose. She also had previously been told in her home country that she had endometriosis solely based on her gynaecological symptoms-menorrhagia, dysmenorrhea and deep dyspareunia. She was not treated empirically with the combined oral contraceptive pill or progestagens. She also complained of neck pain which was investigated with no cause found.

Physical examination was normal. Chest was clear; heart sounds were dual with no murmurs. There was no clubbing and any cervical lymphadenopathy. Abdomen was soft with no palpable masses. She had a normal spirometry.
Blood investigations reported an elevated tumour markers-Ca 125 and Ca19-9. Chest x-ray revealed no abnormalities - pneumothorax or pleural effusion. An abdominal ultrasound suggested a $6.6 \times 4.6 \mathrm{~cm}$ mass centred on the right hemidiaphragm extending into the right hemithorax and invading the right lobe of the liver. She then had CT/MRI scan (Figure 1) and a further PET-CT scan (Figure 2) after discussion at the multidisciplinary departmental meetings.
A

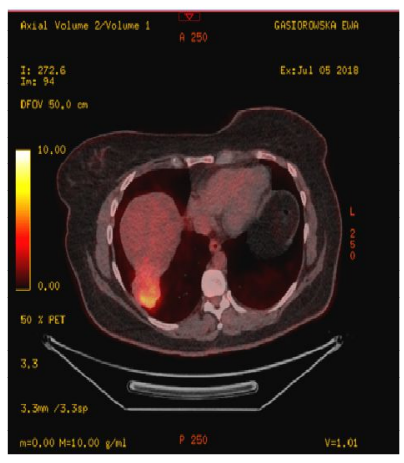

B

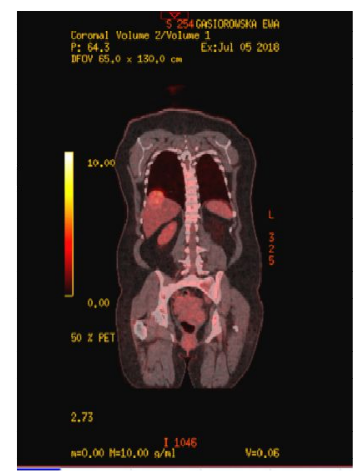

Figure 2: PET 5can. A) The lobulated mass related to the right hemi diaphragm shows a superior component protruding onto the right lower lobe of the lung and $(B)$ a more inferior component protruding into the right lobe of the liver.

The findings were of a $6.5 \mathrm{~cm}$ ill-defined lobulated mass invading the right hemidiaphragm, lower lobe of lung, protruding into the right lobe of the liver (Figure 1). The differential diagnosis was that of the endometriosis or that of a primary pleural malignancy. A liver biopsy was taken
A

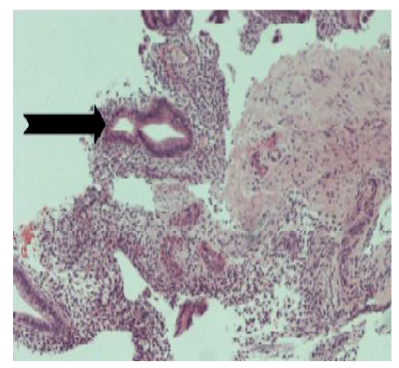

B

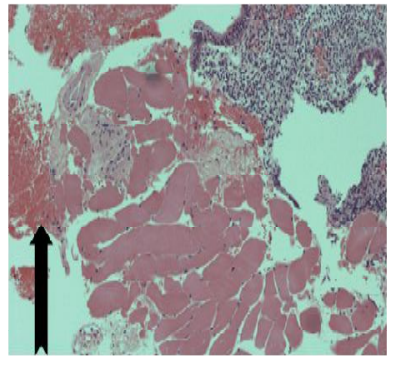

Figure 3: liver Biopsy. A) Berign endometrial tissue and glands with blood, skeletal muscles and fibrous tissue in the background. B|Brown pigment is seen within the stroma, likely representing hemosiderin.

which confirmed hepatic endometriosis. Given that it was a benign diagnosis (Figure 3), she was then seen by the hepatobiliary surgeon who discussed the possibility of a liver 
and or lung resection. After further discussion at the gynaecology multidisciplinary departmental meeting, it was felt that in view of her age and her perimenopausal status a medical therapy approach with gonadotropin-releasing hormone agonist $(\mathrm{GnRH})$ or progestagens rather than resectional surgery would be more feasible. Patient was initially non-compliant to medical therapy and was readmitted to emergency department with severe right upper quadrant pain in relation to her menstruation. She underwent further imaging. A CT scan suggested that the endometrioma in liver has significantly enlarged to $10 \mathrm{~cm}$ compared to $6 \mathrm{cms}$ previously. There was also a ground glass opacification in the right lower lobe of the lung which was a new finding since the previous study (Figure 4- A, B). The patient was counselled, who had then agreed to restart on the GnRH analogues with add-back therapy. In view of her compliance, a CT scan was repeated 3 months later which suggested a smaller endometrioma with reduced density and no further haemorrhage (Figure 4 - C, D).

A

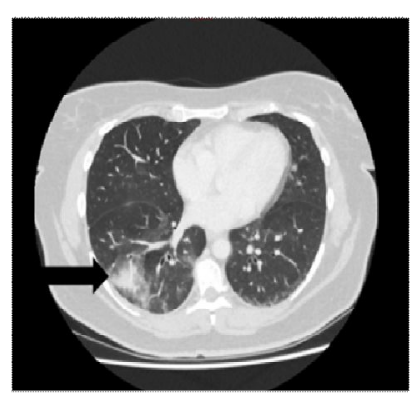

B

$C$

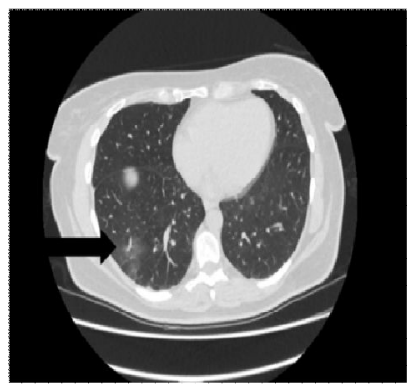

D

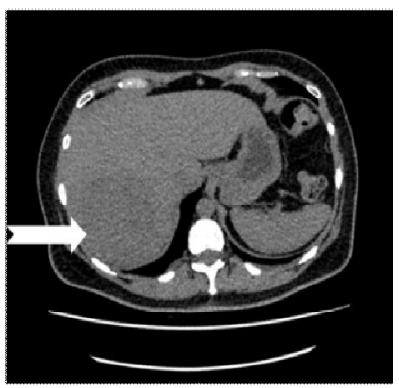

Figure 4: CT scan showing lesions before ( $A$ and $B$ ) and after (C and D) GnRH treatment. A) Ground glass opacification in the right lower lobe of the lung. B) The endometrioma has significantly enlarged in the liver, suggesting substantial haemorrhagic transformation/intralesional bleed. C) Repeated CT scan after 3 months of GnRH treatment showing decreased ground glass opacification in the lower lobe. D) Repeated CT scan after 3 months of GnRH treatment showing smaller endometrioma with reduced density and no further haemorrhage.

At present, she is fully compliant with her medical therapy with complete resolution of her symptoms. She is awaiting further imaging to see the involution of the endometrioma, in the hope that no operative intervention would be required.

\section{Discussion}

Thoracic endometriosis often presents as a delayed diagnosis. The mean age of diagnosis is around 35 years with a range of (19-54 years). This is extrapolated from two large review 1966 and $2010^{5,6}$. The reasons could be partly due to the lack of proper history taking, as multiple symptoms being both cyclical gynaecological and non gynaecolical (dyschezia, dysuria, haematuria, rectal bleeding, shoulder pain) increases the likelihood as having endometriosis or the thoracic component taking a longer time to reveal as the diaphragm undergoes cyclical necrosis and subsequent cycles may lead to the production of fenestrations. Sloughing of the full thickness of the diaphragm (tendinous portionmost frequently involved) by the functioning endometrial tissue could cause coalescence of the smaller fenestrations to produce a larger defect ${ }^{7}$.

On histopathology diaphragmatic endometriosis is characterised by the presence of both endometrial stoma and glands in the form of brown or blue implants with fibrosis, inflammatory infiltrates and haemorrhage.

There are 4 well recognised and distinct clinical entities of thoracic endometriosis: Catamenial pneumothorax, catamenial haemothorax, catamenial haemoptysis and lung nodules. Patients present with catamenial symptoms usually within 24-48 hrs of onset of their menstrual bleed. The most frequent symptoms are chest pain (90\%) followed by dyspnoea (31\%), haemoptysis $(7 \%)$ and rarely cough ${ }^{6}$. Cyclical haemoptysis is extremely rare. A history of Haemoptysis during her menstrual period could be a strong indicator of pulmonary endometriosis. The bleeding can be variable. Symptomatic involvement of the diaphragm by endometriosis is rare and can cause characteristic symptoms. (Ipsilateral chest pain, shoulder, arm and neck pain) that can mainly be aggravated around the time of menstruation. Thoracic endometriosis syndrome (TES) remains an exceedingly rare condition.

Various theories have been proposed to explain the presence of intrathoracic endometrial implants: coelomic metaplasia, lymphatic/haematogenous embolization from the uterus and pelvis and finally retrograde menstruation with transperitoneal and transdiaphragmatic migration of the endometrial cells ${ }^{7}$. The transdiaphragmatic passage could occur either through congenital or acquired defects in the diaphragm. The distribution of the endometrial implants 
through the diaphragm seems to occur in an asymmetrical manner. The right is more commonly affected than the left due to the directional flow of peritoneal fluid from the pelvis along the right paracolic gutter to the subphrenic space. The endometrial tissue located within the peritoneum follows the same directional flow. The falciform ligament then prevents further migration of the tissue to the left side of the diaphragm. Further implantation/colonization and or migration can be facilitated due to the "piston action" of the liver whereby variation in the intraperitoneal pressure with respiration causes the right diaphragm to contract against the liver ${ }^{8}$. In this particular case, full -thickness necrosis with both the visceral and thoracic components led to involvement of the lower lobe lung parenchyma and the right lobe of the liver.

A detailed history (cyclical gynaecological and nongynaecological) taking is the key in the diagnosis of TES. A history of haemoptysis during her menstrual period could be a strong indicator for pulmonary endometriosis as was in this particular case but other pulmonary pathologies such as lung malignancies and tuberculosis and pleural plaques should be considered as they can present with chest pain, haemoptysis, cough and dyspnoea. Clinical examination may reveal an effusion and or a pneumothorax.

Chest X-Ray may reveal an effusion, nodules and a pneumothorax. CT and MRI scans are useful in providing a differential diagnosis. $\mathrm{CT}$ is accurate in identifying diaphragmatic endometriotic lesions, single or multiple pulmonary nodules and a pneumoperitoneum. Chest MRI is superior to CT in view of differentiating pleural from parenchymal implants and is also better at characterisation of haemorrhagic lesions. Video-assisted thoracoscopy allows direct assessment of the lung and the diaphragmatic surfaces. It reveals diaphragmatic perforations, nodular or plaque like endometrial deposits which can be brown or violet in colour in relation to the menstrual cycle or even larger masses ${ }^{7}$. Video assisted laparoscopy has been also been used in patients with catamenial chest pain suggestive of diaphragmatic endometriosis. Bronchoscopy may have a role in localisation of the bleeding lobe or segment of the lung in catamenial haemoptysis. Bronchial biopsy almost constantly fails to provide a tissue diagnosis regardless of the bronchoscopy findings, whereas the brush cytology reveals endometrial cells ${ }^{9}$. Bronchial artery angiography has been used to establish the diagnosis of parenchymal endometriosis in catamenial haemoptysis. It is not diagnostic as normal angiograms have been found in many cases.
Thoracic endometriosis can be medically or surgically treated. Medical therapy consists of suppression of the ectopic endometrial tissue. This can be achieved by the use of oral contraceptive pill, progestogens, danazol or $\mathrm{GnRH}$ analogues. GnRH agonist cause down regulation of the receptors, hence creating a reversible hypogonadotropic hypogonadism. Danazol on the other hand, prevents a midcycle luteinising hormone surge thereby decreasing the estradiol secretion and bringing about anovulation. There is uncertainty regarding the optimal duration of administration of these drugs. In addition there is also a high rate of recurrence after hormonal therapy in about $50 \%$ of cases ${ }^{5}$. A sequential medical-surgical or surgical-medical option may become necessary if one has not achieved a satisfactory response to the first line of treatment. The use of VATSvideo-assisted thoracoscopic surgery has been reserved for symptomatic patients. It allows multiple treatment modalities to be implemented such as resection of both parenchymal and diaphragmatic implants, suturing of the defects, using the endoscopic stapler or even placement of an artificial polygalactin mesh. The drawback is that the endometriosis on the abdominal surface may be missed. Superficial diaphragmatic endometriosis can be treated with cold scissors, monopolar and bipolar energy and $\mathrm{CO}_{2}$ laser therapy. In a case series of 8 symptomatic patients with diaphragmatic endometriosis with a 1-7 year follow up, the author found that laparoscopic diagnosis of diaphragmatic endometriosis followed by laparotomy and full thickness resection of the involved diaphragm resulted in symptoms reduction in $100 \%$ and long - term symptomatic cure in $88 \%$ in the affected patients ${ }^{10}$. Due to the complexity and rarity of the disease there should be a multidisciplinary input in symptomatic patients who have both pelvic and thoracic symptoms. Hysterectomy with bilateral salpingooophorectomy removes the underlying cause but if hormone replacement therapy is initiated there may be then be a recurrence of thoracic endometriosis.

\section{Conclusion}

To our best knowledge and reviewing the literature, we present a rare case report where the patient was found to have a lobulated mass related to the right hemidiaphragm, invading the right lower lobe of the lung and the liver. She presented with catamenial haemoptysis, and long standing right upper quadrant, neck, shoulder and arm pain. Interestingly, she was investigated for recurrent right upper quadrant pain, with no cause found. The diagnosis of diaphragmatic endometriosis is challenging and requires a 
The New Indian Journal of OBGYN. 2020 (July-December); 7(1)

high index of suspicion when patients presents with nongynaecological symptoms related and aggravated by their menstruation. Due to the complexity and rarity of this condition, the diagnosis is often delayed or missed and can have a major impact and a detrimental effect on the patients overall health and fertility.

\section{Conflict of interest: None. Disclaimer: Nil.}

\section{References}

1. Agarwal N, Subramanian A. Endometriosismorphology, clinical presentations and molecular pathology. J Lab Physicians. 2010; 2(1):1-9.

2. Buck Louise G, Hediger ML, Peterson CM, Sundaram $\mathrm{R}$, Stanford J, Chen Z, et al. Incidence of endometriosis by study population and diagnostic method: the ENDO study. Fertil Steril. 2011; 96(2): 360-5.

3. Veeraswamy A, Lewis M, Mann A, Kotikela S , Hajhosseini B , Nezhat C. Extragenital endometriosis. Clin Obstet Gynecol. 2010; 53: 449-66.

4. Honore G. Extrapelvic endometriosis. Clinical Obstet Gynaecol. 1999; 42 :699-711.

5. Joseph J, Sahn SA. Thoracic endometriosis syndrome: new observations from an analysis of 110 cases. Am J Med. 1996; 100:164-70.
6. Channabasavaiah AD, Joseph JV. Thoracic endometriosis: revisiting the association between clinical presentation and thoracic pathology based on thoracoscopic findings in 110 patients. Medicine (Baltimore). 2010; 89(3): 183-6.

7. Alifano M, Trisolini R, Cancellieri A, Regnard JF. Thoracic Endometriosis: current knowledge. Annals of thoracic surgery. 2006; 81:761-9.

8. 8 Kirschner PA. Porous diaphragm syndromes. Chest surg Clin North Am. 1998; 8: 449-72.

9. Kuo PH, Wang HC , Liaw YS , Kuo SH. Bronchoscopic and angiographic findings in tracheobronchial endometriosis . Thorax. 1996; 51: 1060-1.

10. Redwine DB. Diaphragmatic endometriosis diagnosis, surgical management, and long-term results of treatment. Fertil Steril. 2002; 77: 288.

\section{Azal EL Adwan ${ }^{1}$, Harjit Singh Dhaliwal ${ }^{2}$, Samah Ballal ${ }^{3}$,} Rachana Dwivedi ${ }^{4}$

1,2,3,4 Department of Obstetrics and Gynaecology, Royal Bournemouth Hospital, Castle Lane East , Bournemouth, BH7 7DW. 